\title{
Profilaxia para úlcera de estresse na Unidade de Terapia Intensiva: uma revisão integrativa
}

\author{
Stress ulcer prophylaxis in the Intensive Care Unit: an integrative review \\ Profilaxis de úlceras por estrés en la Unidad de Cuidados Intensivos: una revisión integradora
}

Rafael Ferreira da Silva ORCID: https://orcid.org/0000-0002-9084-4332 Mauricio de Nassau, Brasil

E-mail: rafhammenor@gmail.com

Samuel Lopes dos Santos ORCID: https://orcid.org/0000-0003-3375-9171 Universidade Federal do Piaú, Brasil

E-mail: samuellopes121314@gmail.com

Thyago de Oliveira Afonso ORCID: https://orcid.org/0000-0001-7616-9011

Universidade Federal de Pernambuco, Brasil E-mail: thyago.oafonso@gmail.com

Felipe Pereira da Silva

ORCID: https://orcid.org /0000-0002-6489-4096 Centro Universitário de Ciências e Tecnologia do Maranhão, Brasil

E-mail: felipepsilva1@gmail.com

Simone de Sousa Cunha Ribeiro ORCID: https://orcid.org/0000-0002-4117-3034 Centro Universitário UniFacid, Brasil

E-mail: Simonecunha101@hotmail.com

Suhelen Maria Brasil da Cunha Gama

ORCID: https://orcid.org/0000-0002-5273-5426 Universidade Federal do Piaú, Brasil E-mail: suhelen_gama@hotmail.com

Sara da Silva Siqueira Fonseca ORCID: https://orcid.org/0000-0002-2209-5501 Universidade Federal do Piauí, Brasil E-mail: ss.siqueira@hotmail.com

Airton César Leite

ORCID: https://orcid.org/0000-0001-7184-8488 Centro Universitário Santo Agostinho, Brasil E-mail: ainton.cesar2014@gmail.com

Maria da Conceição Viana Sousa ORCID: https://orcid.org/0000-0002-1883-6602

Centro Universitário Santo Agostinho, Brasil E-mail: mariacont208@gmail.com

Marcela Flavia Lopes Barbosa ORCID: https://orcid.org/0000-0001-7061-2342

Universidade Federal do Piauí, Brasil

E-mail:marcelaaflavia@hotmail.com

Francisco Gaunié de Sousa Pessôa ORCID: https://orcid.org/0000-0002-6630-3405 Faculdade do Piauí, Brasil E-mail: gaunie.sousa10@hotmail.com

Gabrielly Martins de Barros

ORCID: https://orcid.org/0000-0001-9696-424X

Universidade Federal do Piauí, Brasil

E-mail: gabriellymb95@gmail.com

Francisco Rafael de Carvalho

ORCID: https://orcid.org/0000-0003-3479-098X

Faculdade Uninassau Aliança, Brasil

E-mail: rafaelcarvalhojose@gmail.com

Aldemes Barroso da Silva

ORCID: https://orcid.org/0000-0002-6677-5648 Universidade Federal do Piaú, Brasil

E-mail: aldemesbarroso@hotmail.com 
Whellyda Katrynne Silva Oliveira

ORCID: https://orcid.org/0000-0003-1920-1454 Universidade Federal do Piauí, Brasi E-mail: oliveirawks@gmail.com

Ronnyely Suerda Cunha Silva

ORCID: https://orcid.org/0000-0003-3710-7824 Universidade Federal do Piauí, Brasi

E-mail: ronnyelynutricionista@gmail.com

Amanda Costa Maciel

Orcid https://orcid.org/0000-0002-2668-037X Universidade Federal de Sergipe, Brasi

E-mail: amandacmaciel@hotmail.com

\begin{abstract}
Resumo
A úlcera de estresse (UE) é uma complicação comum nos pacientes internados em unidades de terapia intensiva (UTI), que são definidas como úlceras do trato gastrointestinal superior (TGIS), sendo que algumas formas podem evoluir com hemorragia. Dadas as consequências hemodinâmicas ocasionadas nos pacientes com UE no contexto de unidade de terapia intensiva, este artigo tem como objetivo investigar causas fisiopatológicas, profilaxias e terapias para evitar riscos de sangramento. Trata-se de um estudo de revisão bibliográfica e exploratório no método revisão integrativa de literatura, realizado nas bases de dados do PUBMED, EMBASE e LILACS, através dos descritores/ palavras-chave: "Úlcera de Estresse" AND "Unidade de Terapia Intensiva". Inicialmente a busca retornou 1.074 trabalhos, após aplicados os critérios de inclusão e exclusão foram selecionados sete para este estudo. Observou-se que pacientes em ventilação mecânica por mais de 48 horas, coagulopatia, choque, insuficiência renal com hemodiálise, passado de úlcera péptica, hemorragia digestiva alta previa, politraumatizado, séptico e insuficiência hepática, possuem risco aumentado em $3600 \%$ para desenvolvimento de UE. A profilaxia deverá ser realizada de forma individualizada e seguindo recomendações específicas para a população local, para evitar erros, uso indevido de antibióticos e retirada prévia inadequada. Ressalta-se que há poucos estudos randomizados que avaliam a eficácia terapêutica da profilaxia da UE no Brasil, necessitando de novos estudos para avaliação direcionada à população brasileira.
\end{abstract}

Palavras-chave: Profilaxia de úlcera de estresse; Terapia intensiva; Cuidado Intensivo.

\title{
Abstract
}

Stress ulcers (SU) are a common complication in patients admitted to intensive care units (ICU), which are defined as upper gastrointestinal tract ulcers, and some forms can evolve to hemorrhage. Given the hemodynamic consequences caused in patients with SU in the context of the intensive care unit, this article aims to investigate pathophysiological causes, prophylaxis and therapies to avoid bleeding risks. This is a bibliographical and exploratory review study using the integrative literature review method, carried out in the PUBMED, EMBASE and LILACS databases, using the descriptors/key words: "Stress Ulcer" AND "Intensive Care Unit". Initially, the search returned 1,074 works, after applying the inclusion and exclusion criteria, seven were selected for this study. It was observed that patients on mechanical ventilation for more than 48 hours, coagulopathy, shock, renal failure with hemodialysis, history of peptic ulcer, previous upper digestive hemorrhage, multiple trauma, septic and liver failure, have an increased risk of $3600 \%$ for developing EU. Prophylaxis should be carried out individually and following specific recommendations for the local population, to avoid errors, misuse of antibiotics and inadequate prior withdrawal. It is noteworthy that there are few randomized studies that assess the therapeutic efficacy of SU prophylaxis in Brazil, requiring further studies for evaluation directed at the Brazilian population.

Keywords: Stress ulcer prophylaxis; Intensive therapy; Intensive Care.

\section{Resumen}

Las úlceras por estrés (UE) son una complicación común en pacientes ingresados en unidades de cuidados intensivos (UCI), que se definen como úlceras del tracto gastrointestinal superior (TGIS), y algunas formas pueden evolucionar a hemorragia. Dadas las consecuencias hemodinámicas provocadas en pacientes con UE en el contexto de la unidad de cuidados intensivos, este artículo tiene como objetivo investigar causas fisiopatológicas, profilaxis y terapias para evitar riesgos hemorrágicos. Se trata de un estudio de revisión bibliográfica y exploratoria mediante el método de revisión integradora de la literatura, realizado en las bases de datos de PUBMED, EMBASE y LILACS, utilizando los descriptores / palabras clave: "Úlcera por estrés" Y "Unidad de Cuidados Intensivos". Inicialmente, la búsqueda arrojó 1.074 trabajos, luego de aplicar los criterios de inclusión y exclusión, siete fueron seleccionados para este estudio. Se observó que los pacientes en ventilación mecánica por más de 48 horas, coagulopatía, shock, insuficiencia renal con hemodiálisis, antecedentes de úlcera péptica, hemorragia digestiva alta previa, trauma múltiple, insuficiencia séptica y hepática, tienen un riesgo aumentado de $3600 \%$ para desarrollar UE. La profilaxis debe realizarse de forma individual y siguiendo recomendaciones específicas para la población local, para evitar errores, mal uso de antibióticos y abstinencia previa inadecuada. Es de destacar que existen pocos estudios aleatorizados que evalúen la eficacia terapéutica de la profilaxis de la UE en Brasil, lo que requiere más estudios de evaluación dirigidos a la población brasileña.

Palavras-clave: Profilaxis de úlceras por estrés; Terapia intensiva; Cuidados intensivos. 


\section{Introdução}

A úlcera de estresse (UE) é uma complicação comum nos pacientes internados em unidades de terapia intensiva (UTI), que são definidas como úlceras do trato gastrointestinal superior (TGIS), sendo que algumas formas podem evoluir com hemorragia. Assim, tais pacientes críticos necessitam de profilaxia primária para prevenir sangramentos pela UE ou tratamento para sangramento relacionado às úlceras (Dargent et al., 2020; Gupta et al., 2020; Mendes et al., 2019; Morgan et al., 2019).

A hemorragia por UE é um sangramento secundário do TGIS, já que ocorre em função da internação do paciente, diferentemente dos casos primários de sangramento do TGIS. É importante salientar que as úlceras com sangramento exteriorizado podem acarretar em alterações hemodinâmicas, que estão relacionadas com a diminuição da hemoglobina ou a necessidade de transfusão de concentrado de hemácias em 24h após o início do sangramento (Mendes et al., 2019; Morgan et al., 2019).

A fisiopatologia através da hipersecreção ácida típica, um fator comum em úlceras duodenais não é o principal mecanismo de patogenia das lesões encontradas em pacientes críticos. No entanto, devido ao contexto de disfunção múltipla orgânica, a redução da capacidade protetora gástrica associada à redução de secreção de muco gástrico e bicarbonato explicam as lesões características. Ademais, em indivíduos sépticos há liberação de mediadores inflamatórios e queda na perfusão esplâncnica, os quais predispõem o paciente às erosões de mucosa gástrica e aumento do risco de sangramento (Mendes et al., 2019).

A incidência da UE varia em função de sua definição, presença de fatores de risco e prescrição da profilaxia. Enquanto que a estimativa para pacientes críticos assintomáticos com UE sem receber profilaxia são de mais de $75 \%$, os números são bem inferiores para pacientes com sangramento oculto, entre 15-50\%, para pacientes com exteriorização do sangramento, entre 1,58,5\%, e para pacientes com sangramento significativo em instabilidade hemodinâmica, entre 1-3\% (Morgan et al., 2019).

Tendo em vista o quadro de incidência e as consequências hemodinâmicas ocasionadas nos pacientes com UE no contexto de unidade de terapia intensiva, este artigo tem como objetivo investigar causas fisiopatológicas, profilaxias e terapias para evitar riscos de sangramento.

\section{Metodologia}

Trata-se de uma estudo de revisão bibliográfica e exploratório no método revisão integrativa de literatura, realizado entre os meses de junho e julho de 2021, com abordagem descritiva, nas bases de dados do Sistema Online de Busca e Análise de Literatura Médica (Medical Literature Analysis and Retrieval System Online) da Biblioteca Nacional de Medicina dos Estados Unidos da América (US National Library of Medicine - NLM) - MEDLINE/PubMed, Banco de Dados Bibliográficos Biomédico e Farmacológico (Excerpta Medica dataBASE) - EMBASE e Literatura Latino-americana e do Caribe em Ciências da Saúde - LILACS, através dos descritores/ palavras-chave: "Úlcera de Estresse" AND "Unidade de Terapia Intensiva", combinados com o operador booleano "AND". Tratando-se de uma pesquisa exploratória e descritiva dos dados, assumindo uma gama de conhecimento, fenômenos e problemas, com o objetivo principal de descrever e quantificar a natureza das variáveis (Koche, 2011).

Para execução deste estudo, foram seguidas as etapas de pesquisa: 1- Elaboração de pergunta norteadora; 2- Busca ou amostragem na literatura; 3- Coleta de dados; 4- Análise crítica dos estudos incluídos; 5- Discussão dos resultados; 6Apresentação dos resultados em revisão integrativa (Koche, 2011; Pereira et al., 2018).

Para responder à pergunta norteadora: "Qual a fisiopatologia, profilaxia e terapia para pacientes com úlcera de estresse em Unidade de Terapia Intensiva?", foi realizada busca de artigos nas bases de dados da MEDLINE/Pubmed, EMBASE e Literatura Latino-Americana e do Caribe em Ciências da Saúde (LILACS), com os descritores/MESH: "Úlcera de Estresse" 
AND "Unidade de Terapia Intensiva", utilizando o operador booleano AND. Os critérios de inclusão foram artigos originais disponíveis na íntegra, completos, publicados entre 2017 e junho de 2021, identificados entre 10 de junho e 10 de julho de 2021, nos idiomas português, inglês e/ou espanhol, e temática condizente. Foram excluídos artigos incompletos, cartas, editoriais, duplicados, sem relação com o tema, em pre-proof, teses, dissertações, monografias e manuais.

Após a seleção, foram avaliados os resumos e títulos a fim de identificar a temática condizente para este estudo. Nos casos em que a leitura não foi suficiente para definir a inclusão do artigo na amostra, foram considerados os demais critérios. A elegibilidade foi determinada pela leitura na íntegra dos artigos selecionados. Por fim, os dados referentes ao fluxograma do processo de identificação e seleção dos artigos estão sistematicamente demonstrados na Figura 01.

\section{Resultados}

A busca inicial nas bases de dados a partir dos descritores/MESH retornou 1.074 trabalhos, em sua maior parte obtida do MEDLINE/Pubmed ( $\mathrm{n}=120)$, seguido de EMBASE $(\mathrm{n}=106)$ e LILACS $(\mathrm{n}=2)$. Deste total, após aplicados os critérios de inclusão e exclusão foram selecionados 17 trabalhos para leitura na íntegra, entretanto, após aplicados os critérios de elegibilidade, sete foram aprovados para este estudo, como esquematizado na Figura 1.

Figura 1: Fluxograma de processo de identificação e seleção de artigos.

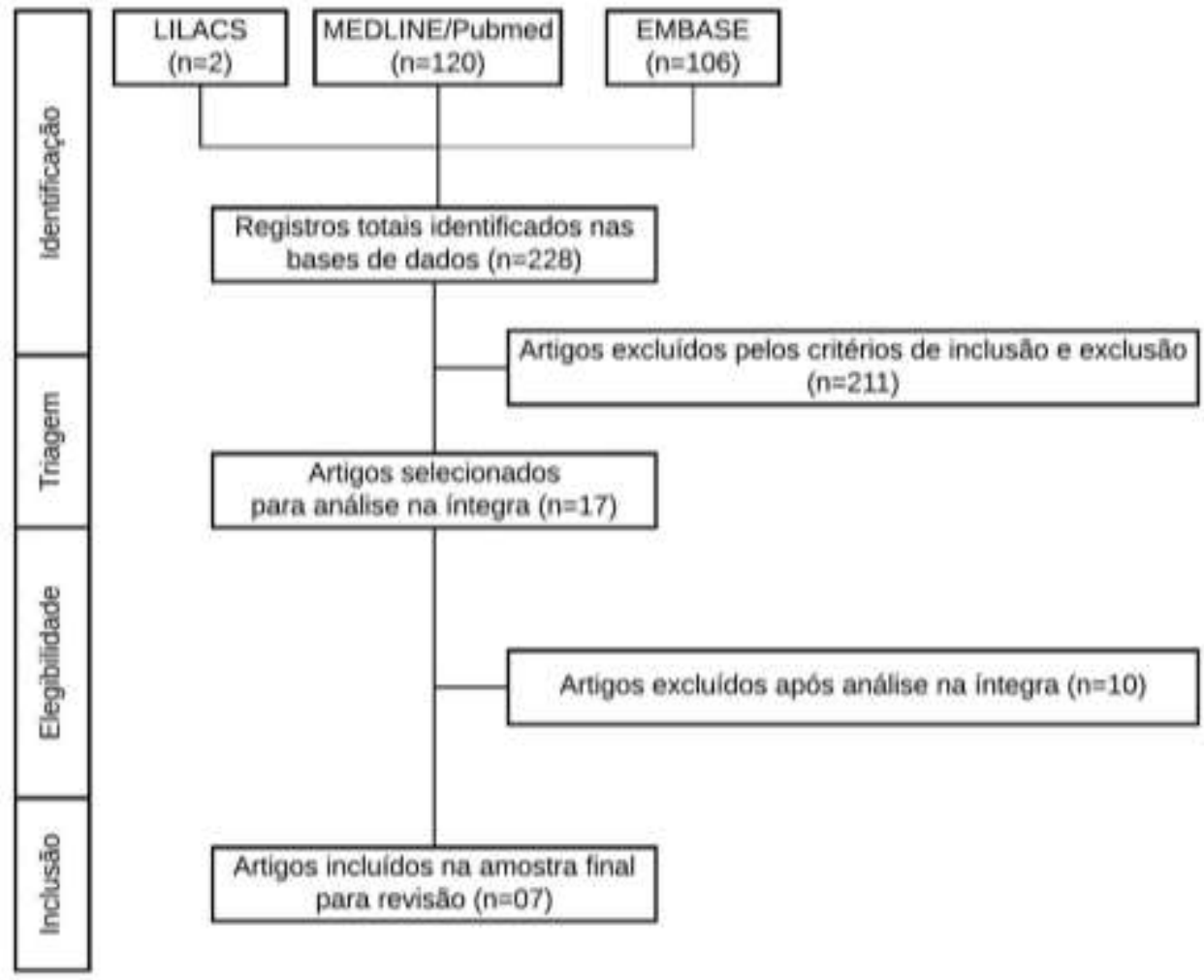

Fonte: Autores

Foram selecionados sete artigos, sendo um estudo retrospectivo, um estudo observacional e cinco ensaios clínicos randomizados, realizados em centros diferentes e produzidos desde 2017 a 2021. Quanto ao idioma, a maioria foi publicado em língua inglesa. As variáveis analisadas para estes artigos foram autor/ano da publicação, título, desenho de estudo, população observada, objetivos do estudo e desfecho/conclusão obtida, como apresentado no Quadro 1. 
Quadro 1: Caracterização dos estudos incluídos na revisão de literatura segundo autores/ano da publicação, título do artigo, desenho do estudo, população observada, objetivos do estudo e desfecho/conclusão.

\begin{tabular}{|c|c|c|c|c|c|}
\hline $\begin{array}{l}\text { Autor/Ano da } \\
\text { publicação }\end{array}$ & Título & $\begin{array}{l}\text { Desenho do } \\
\text { estudo }\end{array}$ & $\begin{array}{l}\text { População } \\
\text { observada }\end{array}$ & Objetivos do estudo & Desfecho/ conclusão \\
\hline $\begin{array}{l}\text { Santos et al., } \\
2020 .\end{array}$ & $\begin{array}{l}\text { Adesão a um } \\
\text { protocolo de } \\
\text { profilaxia de úlcera de } \\
\text { estresse em pacientes } \\
\text { críticos: estudo de } \\
\text { coorte prospectiva }\end{array}$ & $\begin{array}{l}\text { Coorte } \\
\text { prospectivo }\end{array}$ & $\begin{array}{l}\text { Pacientes adultos } \\
\text { admitidos às unidades } \\
\text { de terapia intensiva } \\
\text { clínica e cirúrgica de } \\
\text { um hospital terciário } \\
\text { acadêmico. }\end{array}$ & $\begin{array}{l}\text { Avaliar a adesão ao } \\
\text { protocolo de profilaxia de } \\
\text { úlcera de estresse em } \\
\text { pacientes críticos de um } \\
\text { hospital universitário } \\
\text { terciário. }\end{array}$ & $\begin{array}{l}\text { Foram incluídos } 234 \text { pacientes no período compreendido entre } 2 \text { de julho e } 31 \text { de } \\
\text { julho de } 2018 \text {. Os pacientes tinham idade de } 52 \pm 20 \text { anos, sendo } 125 \text { ( } 53 \% \text { ) deles } \\
\text { cirúrgicos, e o SAPS } 3 \text { médio foi de } 52 \pm 20 \text {. O total de uso apropriado de profilaxia } \\
\text { de úlcera de estresse foi de } 64 \% \text {. Fatores associados com prescrição adequada de } \\
\text { profilaxia de úlcera de estresse foram ventilação mecânica, com RC } 2,13 \text { (IC } 95 \% \\
1,64 \text { - 2,75), e coagulopatia, com RC } 2,77 \text { (IC95\% } 1,66-4,60 \text { ). A incidência de } \\
\text { sangramento do trato gastrointestinal superior foi de } 12,8 \% \text {. A adesão ao protocolo } \\
\text { de profilaxia de úlcera de estresse foi baixa, e o uso inadequado de profilaxia de } \\
\text { úlcera de estresse foi frequente nesta coorte de pacientes críticos. }\end{array}$ \\
\hline $\begin{array}{l}\text { Franchitti et } \\
\text { al., } 2020 .\end{array}$ & $\begin{array}{l}\text { Adequacy of stress } \\
\text { ulcer prophylaxis } \\
\text { prescription in the } \\
\text { intensive care unit: an } \\
\text { observational study }\end{array}$ & $\begin{array}{l}\text { Observacion } \\
\text { al }\end{array}$ & $\begin{array}{l}\text { Todos os pacientes } \\
\text { sem indicação prévia } \\
\text { de terapia supressora } \\
\text { e internados em } \\
\text { Unidade de Terapia } \\
\text { Intensiva durante } 24 \mathrm{~h} \\
\text { a } 2 \text { meses. }\end{array}$ & $\begin{array}{l}\text { Analisar a adequação da } \\
\text { profilaxia para úlcera em } \\
\text { UTI e quantificar a } \\
\text { proporção de pacientes } \\
\text { que receberam profilaxia } \\
\text { de úlcera de estresse } \\
\text { inadequada após alta da } \\
\text { UTI. }\end{array}$ & $\begin{array}{l}\text { Foram admitidos } 372 \text { pacientes durante o período do estudo, } 140 \text { preencheram os } \\
\text { critérios de inclusão. Destes, } 130 \text { receberam profilaxia para úlcera de estresse na } \\
\text { UTI. A profilaxia consistiu de esomeprazol em } 86,2 \% \text { dos pacientes. No geral, a } \\
\text { profilaxia da UE foi inadequada em } 65,3 \% \text { dos pacientes. Na alta da UTI, a profilaxia } \\
\text { foi mantida errada em } 51,9 \% \text { dos pacientes. Da mesma forma, a terapia pós alta } \\
\text { hospitalar foi mantida de forma errada em } 28 \% \text { dos pacientes. }\end{array}$ \\
\hline $\begin{array}{l}\text { Muzlovic et } \\
\text { al., } 2019\end{array}$ & $\begin{array}{l}\text { Stress ulcer } \\
\text { prophylaxis as a risk } \\
\text { factor for tracheal } \\
\text { colonization and } \\
\text { hospital-acquired } \\
\text { pneumonia in } \\
\text { intensive care } \\
\text { patients: impact on } \\
\text { latency time for } \\
\text { pneumonia. }\end{array}$ & $\begin{array}{l}\text { Ensaio } \\
\text { clínico } \\
\text { randomizado }\end{array}$ & $\begin{array}{l}\text { Todos os pacientes } \\
\text { em Unidade de } \\
\text { Terapia Intensiva que } \\
\text { estavam } \\
\text { hospitalizados, } \\
\text { traqueostomizados ou } \\
\text { em ventilação } \\
\text { mecânica que } \\
\text { receberam profilaxia } \\
\text { para UE. }\end{array}$ & $\begin{array}{l}\text { Avaliar pacientes em uso } \\
\text { de profilaxia de UE com } \\
\text { maior risco de } \\
\text { sangramento e pneumonia } \\
\text { e determinar fatores que } \\
\text { influenciam no processo } \\
\text { hemodinâmico. }\end{array}$ & $\begin{array}{l}\text { Fatores de risco que pioraram significativamente o resultado da doença também } \\
\text { aumentaram a mortalidade. Dentre os fatores de risco elencados, o de maior } \\
\text { importância foi a presença de doença cardíaca e circulatória como doença de base, } \\
\text { além do uso prévio de antibióticos na presença de ventilação mecânica. O artigo } \\
\text { sugere a prevenção precoce de infecções hospitalares e o uso racional de antibióticos. }\end{array}$ \\
\hline $\begin{array}{l}\text { Krag et al., } \\
2017\end{array}$ & $\begin{array}{l}\text { Stress ulcer } \\
\text { prophylaxis in the } \\
\text { intensive care unit } \\
\text { trial: detailed } \\
\text { statistical analysis } \\
\text { plan }\end{array}$ & $\begin{array}{l}\text { Estudo } \\
\text { clínico } \\
\text { randomizado } \\
\text { multicêntrico } \\
\text { e duplo cego }\end{array}$ & $\begin{array}{l}3.350 \text { pacientes } \\
\text { adultos de UTI com } \\
\text { doença aguda e risco } \\
\text { de sangramento } \\
\text { gastrointestinal }\end{array}$ & $\begin{array}{l}\text { Avaliar os benefícios e } \\
\text { danos da profilaxia de } \\
\text { úlcera de estresse com um } \\
\text { inibidor da bomba de } \\
\text { prótons em pacientes } \\
\text { adultos na unidade de }\end{array}$ & $\begin{array}{l}\text { O desfecho primário foi a mortalidade em } 90 \text { dias. Os desfechos secundários incluem } \\
\text { a proporção de pacientes com sangramento gastrointestinal clinicamente importante, } \\
\text { pneumonia, infecção por Clostridium difficile ou isquemia do miocárdio, dias vivos } \\
\text { sem suporte de vida, reações adversas graves, mortalidade em } 1 \text { ano. }\end{array}$ \\
\hline
\end{tabular}




\begin{tabular}{|c|c|c|c|c|c|}
\hline & & & & terapia intensiva (UTI). & \\
\hline $\begin{array}{l}\text { Krag et al., } \\
2018\end{array}$ & $\begin{array}{l}\text { Pantoprazole in } \\
\text { patients at risk for } \\
\text { gastrointestinal } \\
\text { bleeding in the ICU. }\end{array}$ & $\begin{array}{l}\text { Estudo } \\
\text { clínico } \\
\text { randomizado } \\
\text { multicêntrico } \\
\text { e duplo cego }\end{array}$ & $\begin{array}{l}\text { Um total de } 3.298 \\
\text { pacientes foram } \\
\text { inscritos; } 1645 \text { foram } \\
\text { atribuídos } \\
\text { aleatoriamente ao } \\
\text { grupo pantoprazol e } \\
1653 \text { ao grupo } \\
\text { placebo. }\end{array}$ & $\begin{array}{l}\text { Avaliar os riscos e } \\
\text { benefícios da profilaxia } \\
\text { com pantoprazol para } \\
\text { pacientes com UE em UTI }\end{array}$ & $\begin{array}{l}\text { Os dados sobre o desfecho primário estavam disponíveis para } 3.282 \text { pacientes } \\
(99,5 \%) \text {. Aos } 90 \text { dias, } 510 \text { pacientes }(31,1 \%) \text { no grupo pantoprazol e } 499 \text { (30,4\%) no } \\
\text { grupo placebo morreram (risco relativo, } 1,02 \text {; intervalo de confiança de } 95 \% \text { [IC], } \\
0,91-1,13 ; \mathrm{P}=0,76) \text {. Durante a permanência na UTI, pelo menos um evento } \\
\text { clinicamente importante (um composto de sangramento gastrointestinal clinicamente } \\
\text { importante, pneumonia, infecção por Clostridium difficile ou isquemia do miocárdio) } \\
\text { ocorreu em } 21,9 \% \text { dos pacientes designados para pantoprazol e } 22,6 \% \text { daqueles } \\
\text { designados para placebo (relativo risco, } 0,96 ; \text { IC } 95 \%, 0,83-1,11) \text {. No grupo do } \\
\text { pantoprazol, } 2,5 \% \text { dos pacientes apresentaram sangramento gastrointestinal } \\
\text { clinicamente importante, em comparação com } 4,2 \% \text { no grupo do placebo. O número } \\
\text { de pacientes com infecções ou reações adversas graves e a porcentagem de dias vivos } \\
\text { sem suporte de vida em } 90 \text { dias foram semelhantes nos dois grupos. }\end{array}$ \\
\hline $\begin{array}{l}\text { Schefold et } \\
\text { al., } 2019\end{array}$ & $\begin{array}{l}\text { Outcomes of } \\
\text { Prophylactic } \\
\text { Pantoprazole in Adult } \\
\text { Intensive Care Unit } \\
\text { Patients Receiving } \\
\text { Dialysis: Results of a } \\
\text { Randomized Trial }\end{array}$ & $\begin{array}{l}\text { Estudo } \\
\text { clínico } \\
\text { randomizado }\end{array}$ & $\begin{array}{l}3291 \text { pacientes de } \\
\text { unidade de terapia } \\
\text { intensiva (UTI) com } \\
\text { lesão renal aguda que } \\
\text { requerem terapia } \\
\text { renal substitutiva } \\
\text { (TRS) }\end{array}$ & $\begin{array}{l}\text { Avaliar a incidência de } \\
\text { sangramento } \\
\text { gastrointestinal e os } \\
\text { efeitos da profilaxia de } \\
\text { úlcera por estresse nos } \\
\text { pacientes hospitalizados } \\
\text { em UTIs com lesão renal } \\
\text { aguda que requerem } \\
\text { terapia renal substitutiva }\end{array}$ & $\begin{array}{l}\text { Foram analisados dados de } 3.291 \text { pacientes adultos internados agudamente na UTI } \\
\text { com um ou mais fatores de risco para sangramento gastrointestinal randomizados } \\
\text { para pantoprazol ou placebo por via intravenosa uma vez ao dia durante a internação } \\
\text { na UTI (até a alta da UTI, morte ou um máximo de } 90 \text { dias). Cerca de } 20 \text { de } 258 \\
\text { ( } 7,8 \% \text {, IC de } 95 \% \text { 4,5-11,1\%) e } 52 \text { de } 568 \text { (9,2\%, IC de } 95 \% \text { 6,8-11,6\%) dos } \\
\text { pacientes que receberam TRS no início do estudo e a qualquer momento na UTI, } \\
\text { respectivamente, desenvolveu sangramento gastrointestinal clinicamente importante } \\
\text { na UTI. Não observamos diferenças estatisticamente significativas no efeito da } \\
\text { intervenção (pantoprazol vs. placebo) na proporção de pacientes com sangramento } \\
\text { GI clinicamente importante, eventos clinicamente importantes, eventos adversos } \\
\text { infecciosos, uso de intervenções para parar o sangramento GI ou mortalidade em } 90 \\
\text { dias em pacientes com versus sem TRS no início do estudo. }\end{array}$ \\
\hline $\begin{array}{l}\text { Marker et al., } \\
2019\end{array}$ & $\begin{array}{l}\text { Pantoprazole in ICU } \\
\text { patients at risk for } \\
\text { gastrointestinal } \\
\text { bleeding-1-year } \\
\text { mortality in the SUP- } \\
\text { ICU trial }\end{array}$ & $\begin{array}{l}\text { Estudo } \\
\text { clínico } \\
\text { randomizado }\end{array}$ & $\begin{array}{l}\text { Pacientes adultos } \\
\text { internados } \\
\text { agudamente na UTI } \\
\text { com risco de } \\
\text { sangramento } \\
\text { gastrointestinal }\end{array}$ & $\begin{array}{l}\text { Avaliar a mortalidade em } \\
1 \text { ano e fazer análises de } \\
\text { sensibilidade de acordo } \\
\text { com o protocolo do ensaio } \\
\text { e o plano de análise } \\
\text { estatística. }\end{array}$ & $\begin{array}{l}\text { Um total de } 3.261 \text { dos } 3.291 \text { pacientes com dados disponíveis }(99,1 \%) \text { foram } \\
\text { acompanhados em } 1 \text { ano após a randomização; } 1635 \text { foram atribuídos ao pantoprazol } \\
\text { e } 1626 \text { ao placebo. Em } 1 \text { ano após a randomização, } 610 \text { de } 1.635 \text { pacientes }(37,3 \%) \\
\text { morreram no grupo de pantoprazol em compação com } 601 \text { de } 1.626(37,0 \%) \text { no } \\
\text { grupo de placebo (risco relativo, } 1,01 \text {; intervalo de confiança de } 95 \% \text { 0,92-1,10). Os } \\
\text { resultados foram consistentes na análise de sensibilidade ajustada para fatores de } \\
\text { risco da linha de base e na população por protocolo. Não se observou } \\
\text { heterogeneidade no efeito do pantoprazol vs placebo na mortalidade de } 1 \text { ano nos } \\
\text { subgrupos predefinidos, ou seja, pacientes com e sem choque, ventilação mecânica, } \\
\text { doença hepática, coagulopatia, alta gravidade da doença (SAPS II> 53) ou em } \\
\text { pacientes de UTI médica versus cirúrgica. }\end{array}$ \\
\hline
\end{tabular}




\section{Discussão}

A úlcera por estresse (UE) ocorre principalmente no fundo e no corpo gástrico, com tendência a serem rasas e causando sangramento dos capilares superficiais. No entanto, podem ocorrer lesões mais profundas, com lesões à camada da submucosa e ocasionando hemorragias maciças e, em piores desfechos, perfurações (Mendes et al., 2019; Morgan et al., 2019).

Na maioria dos pacientes, as UE se desenvolvem em regiões proximais do estômago horas após o trauma ou agravamento do paciente, mas apenas uma pequena parcela dos pacientes desenvolve sangramento evidente. Diferentemente, as UE que se desenvolvem em um quadro mais distante da admissão do paciente em terapia intensiva tendem a ser mais profundas e mais distais (Mendes et al., 2019).

Atualmente, não se sabe a distinção entre o desenvolvimento das úlceras precoces e tardias. Porém, independentemente, os dois tipos são ocasionados pelo desbalanço entre os mecanismos de defesa e de produção de ácido gástrico, os quais estão diretamente correlacionados com fatores de risco presentes em cada paciente (Santos et al., 2020; Young et al., 2020).

Vários estudos analisam fatores de risco para o aumento do risco de UE, os quais possuem maior impacto com manifestações clínicas quando acometidos em em unidade de terapia intensiva (UTI) (Mendes et al., 2019; Morgan et al., 2019; Muzlovic et al., 2019). De acordo com Gupta e colaboradores (2020), os fatores de risco são pacientes em choque, insuficiência renal com hemodiálise, passado de úlcera péptica, passado de hemorragia digestiva alta, três ou mais comorbidades, uso de ventilação mecânica, politraumatizado, transplantados, queimados com mais de $35 \%$ do corpo acometido, em uso de antiplaquetários, uso de anti-inflamatórios não esteroidais, insuficiência hepática e sepse.

Reafirmando esse maior risco a UE, os estudos de Muzlovic e colaboradores (2019) e Krag e colaboradores (2017), apontam que pacientes com pelo menos dois fatores de risco, apresentaram uma incidência maior a UE, com valores acima de 3,7\% em comparação com $0,1 \%$ em pacientes sem nenhum fator de risco. Ademais, outros fatores como uso de terapia com glicocorticóides, infecção pelo Helicobacter pylori e uso inadequado de antibióticos podem aumentar essa incidência e piorar o prognóstico do paciente (Franchitti et al., 2020; Krag et al., 2018; Kompas, 2019; Marker et al., 2019).

Enquanto no passado todos os pacientes admitidos na UTI eram tratados com profilaxia para UE, atualmente, com o desenvolvimento de novos estudos e guidelines, o tratamento é indicado apenas para aqueles com risco aumentado de sangramento, baseado em estudos randomizados que demonstraram redução na taxa de sangramento (Barbateskovic et al., 2019; Dargent et al., 2020; Franchitti et al., 2020). Ainda é incerto a caracterização de elevado risco de sangramento, entretanto alguns parâmetros são apresentados para designação:

\section{Critérios para classificação dos pacientes}

Dois ou mais critérios menores: sepse, internamento em UTI por mais de uma semana, sangramento oculto por pelo menos 6 dias e corticoide na terapia;

Trauma crânio encefálico, trauma medular ou queimados;

Passado de úlcera gastrointestinal ou de hemorragia digestiva alta no último ano;

Ventilação mecânica por mais de 48 horas;

Uso de AINEs e de antiplaquetários;

Pacientes com diátese hemorrágica. 
Após avaliação individualizada do paciente em UTI, a escolha da profilaxia deverá ser feita seguindo parâmetros estabelecidos por diretrizes locais ou seguindo recomendações. Cabe ressaltar que a escolha da profilaxia deverá ser avaliada pela equipe multiprofissional, não existindo um agente ideal para o tratamento contra UE em pacientes críticos (Reynalds; MacLaren, 2019; Santos et al., 2020). No entanto, algumas recomendações nacionais e internacionais que foram analisadas nos artigos selecionados afirmam que:

Para os pacientes que podem receber medicação por via enteral, inibidores de bomba de prótons (IBP) são preferíveis ao invés de outros agentes como bloqueadores de receptor de histamina 2 (BRH2), sucralfato ou antiácidos (Krag et al., 2018; Marker et al., 2019; Schefold et al., 2019);

Nos pacientes que não podem receber IBP por via oral, os BRH2 intravenosos podem ser alternativas indicadas, além de possuírem um menor custo. No entanto, a superioridade terapêutica comparada aos IBPs ainda é incerta (Franchitti et al., 2020);

Quando IBP e BRH2 não podem ser administrados, o uso de sucralfato é uma alternativa pela via oral;

O uso de antibióticos deverá ser sempre avaliado e reavaliado individualmente para não causar UE e resistência bacteriana, os quais impactam no pior prognóstico para o paciente (Santos et al., 2020; Muzlovic et al., 2019.

Com relação a duração da profilaxia, estudos apontam que deverá ser realizada até o momento em que o paciente não possuir risco de evoluir com UE. Conduto, ainda não existem parâmetros para essa indicação. Alguns especialistas apoiam que deverá ser retirada a profilaxia quando o paciente não for mais crítico e receber alta da UTI, a não ser que os fatores de risco se mantenham, como coagulopatia ou presença de sangue oculto nas fezes. No entanto, contrapondo esta afirmação, o estudo de Schefold e colaboradores (2019) aponta que pacientes que mantiveram a profilaxia contra UE após alta da UTI tiveram menores recidivas, quando comparados com o mesmo perfil de pacientes com profilaxia cessada (Franchitti et al., 2020; Krag et al., 2018; Kompas, 2019; Marker et al., 2019).

Dados contrapontos e especificidades populacionais, há a necessidade constante de estudos direcionados para estabelecimento de critérios mais precisos para considerar uma profilaxia de UE como padrão. Sendo assim, ressaltamos que há poucos artigos publicados nacionalmente sobre o tema para termos parâmetros mais confiáveis, necessitando, portanto, de outros estudos randomizados e duplo cego para avaliação de eficácia da profilaxia de UE na população brasileira.

\section{Conclusão}

Dada a incidência de UE em pacientes em Terapia Intensiva e quadro agravado por fatores de risco, como pacientes em ventilação mecânica por mais de 48 horas, coagulopatia, choque, insuficiência renal com hemodiálise, passado de úlcera péptica, hemorragia digestiva alta previa, politraumatizado, séptico e insuficiência hepática, observou-se que na totalidade dos artigos analisados, a presença de dois ou mais fatores de risco aumentou $3600 \%$ a chance de um paciente crítico desenvolver UE.

É visto também que a prescrição da profilaxia deverá ser realizada de forma individualizada e seguindo recomendações específicas para a população local, para evitar erros, uso indevido de antibióticos e retirada prévia inadequada.

Ressalta-se que há poucos estudos randomizados que avaliam a eficácia terapêutica da profilaxia da UE no Brasil, o que pode gerar erros na implementação e terapêutica aplicada da profilaxia de UE na população 
brasileira. Sendo assim, faz-se necessários novos estudos de avaliação direcionados à população brasileira para maior compreensão e análise da profilaxia de UE no Brasil.

\section{Referências}

Barbateskovic, M., Marker, S., Granholm, A., Anthon, C. T., Krag, M., Jakobsen, J. C., ... \& Møller, M. H. (2019). Stress ulcer prophylaxis with proton pump inhibitors or histamin-2 receptor antagonists in adult intensive care patients: a systematic review with meta-analysis and trial sequential analysis. Intensive care medicine, 45(2), 143-158.

Blackett, J. W., Faye, A. S., Phipps, M., Li, J., Lebwohl, B., \& Freedberg, D. E. (2020, December). Prevalence and Risk Factors for Inappropriate Continuation of Proton Pump Inhibitors After Discharge From the Intensive Care Unit. In Mayo Clinic Proceedings. Elsevier.

Dargent, A., Jacquier, M., Rozencwajg, S., Andreu, P., \& Quenot, J. P. (2020). Stress ulcer prophylaxis in ICU patients: Answers and questions from the PEPTIC trial.

Franchitti, M., Piubellini, J., Sadeghipour, F., Eckert, P., Voirol, P., \& Schneider, A. G. (2020). Adequacy of stress ulcer prophylaxis prescription in the intensive care unit: an observational study. Swiss Medical Weekly, 150(3536).

Gupta, D., Bhalotra, A. R., \& Singh, R. (2020). Audit on practices of stress ulcer prophylaxis in intensive care unit patients. Indian Journal of Critical Care Medicine: Peer-reviewed, Official Publication of Indian Society of Critical Care Medicine, 24(3), 160.

Koche, J.C. (2011). Fundamentos de metodologia científica: teoria da ciência e iniciação à pesquisa. 1 ed. Petrópolis, RJ: Vozes

Klompas, M. (2019, August). Prevention of intensive care unit-acquired pneumonia. In Seminars in respiratory and critical care medicine (Vol. 40, No. 04, pp. 548-557). Thieme Medical Publishers.

Krag, M., Perner, A., Wetterslev, J., Lange, T., Wise, M. P., Borthwick, M., ... \& Møller, M. H. (2017). Stress ulcer prophylaxis in the intensive care unit trial: detailed statistical analysis plan. Acta Anaesthesiologica Scandinavica, 61(7), 859-868.

Krag, M., Marker, S., Perner, A., Wetterslev, J., Wise, M. P., Schefold, J. C., ... \& Møller, M. H. (2018). Pantoprazole in patients at risk for gastrointestinal bleeding in the ICU. New England Journal of Medicine, 379(23), 2199-2208.

Marker, S., Krag, M., Perner, A., Wetterslev, J., Lange, T., Wise, M. P., ... \& SUP-ICU trial investigators. (2019). Pantoprazole in ICU patients at risk for gastrointestinal bleeding - 1 year mortality in the SUP - ICU trial. Acta Anaesthesiologica Scandinavica, 63(9), 1184-1190.

Marker, S., Perner, A., Wetterslev, J., Barbateskovic, M., Jakobsen, J. C., Krag, M., ... \& Møller, M. H. (2017). Stress ulcer prophylaxis versus placebo or no prophylaxis in adult hospitalised acutely ill patients-protocol for a systematic review with meta-analysis and trial sequential analysis. Systematic reviews, 6(1), 1-8.

Mendes, J. J., Silva, M. J., Miguel, L. S., Gonçalves, M. A., Oliveira, M. J., Oliveira, C. D. L., \& Gouveia, J. (2019). Sociedade Portuguesa de Cuidados Intensivos guidelines for stress ulcer prophylaxis in the intensive care unit. Revista Brasileira de terapia intensiva, 31 (1), 5-14.

Morgan, D. J., Dhruva, S. S., Coon, E. R., Wright, S. M., \& Korenstein, D. (2019). 2019 update on medical overuse: a review. JAMA internal medicine, 179(11), 1568-1574.

Muzlovič, I., \& Štubljar, D. (2019). Stress ulcer prophylaxis as a risk factor for tracheal colonization and hospital-acquired pneumonia in intensive care patients: impact on latency time for pneumonia. Acta Clinica Croatica, 58(1), 72.

Pereira, A. S., Shitsuka, D.M., Parreira,F. J.,\& Shitsuka, R.(2018).Metodologia da pesquisa científica.[e-book]. Santa Maria. Ed (pp. 3-9). UAB/NTE/UFSM. https://repositorio. ufsm. br/bitstream/handle/1/15824/Lic_Computacao_Metodologia-Pesquisa -Cientifica. pdf.

Reynolds, P. M., \& MacLaren, R. (2019). Reevaluating the Utility of Stress Ulcer Prophylaxis in the Critically Ill Patient: A Clinical ScenarioBased MetaAnalysis. Pharmacotherapy: The Journal of Human Pharmacology and Drug Therapy, 39(3), 408-420.

Santos, Y. D. A. P. D., Younes-Ibrahim, M. S., Crozatti, L. L., Raglione, D., Cardozo, L. C. M., Besen, B. A. M. P., ... \& Mendes, P. V. (2020). Adesão a um protocolo de profilaxia de úlcera de estresse em pacientes críticos: estudo de coorte prospectiva. Revista Brasileira de Terapia Intensiva, 32, 37-42.

Schefold, J. C., Krag, M., Marker, S., Perner, A., Wetterslev, J., Wise, M. P., ... \& Møller, M. H. (2019). Outcomes of prophylactic pantoprazole in adult intensive care unit patients receiving dialysis: results of a randomized trial. American journal of nephrology, 50(4), 312-319.

Sun, J., Wang, B., Cao, P., Zhu, H., Lu, K., Geng, P., \& Tan, D. (2019). Benefits and risks of stress ulcer prevention with proton pump inhibitors for critical patients: an observational cohort study with 1972 patients. Zhonghua wei zhong bing ji jiu yi xue, 31(5), 539-544.

Young, P. J., Bagshaw, S. M., Forbes, A. B., Nichol, A. D., Wright, S. E., Bailey, M., ... \& Rowan, K. M. (2020). Effect of stress ulcer prophylaxis with proton pump inhibitors vs histamine-2 receptor blockers on in-hospital mortality among ICU patients receiving invasive mechanical ventilation: the PEPTIC randomized clinical trial. Jama, 323(7), 616-62 